\title{
Selected Properties of Gold
}

Dr. J. Gunther Cohn of Engelhard Industries Division, Engelhard Minerals \& Chemicals Corporation, Menlo Park, New Jersey, U.S.A., has carefully selected from the literature the most generally accepted values for various properties of gold to provide a set of reference data. These pages will form a useful and convenient summary of constants and established values associated with gold.

Nuclear Properties $(1,2)$

\begin{tabular}{|c|c|c|c|c|c|}
\hline \multirow{4}{*}{$\begin{array}{l}\text { Atomic number } \\
\text { Stable isotope, } \mathrm{g} / \text { atom } \\
\text { Selected radioactive } \\
\text { isotopes }\end{array}$} & \multicolumn{3}{|c|}{ Numerical value } & Ref. & Notes \\
\hline & & 79 & & & \\
\hline & & 197 & & & \\
\hline & $\begin{array}{l}\text { Mass } \\
191 \\
192 \\
193 \\
194 \\
195 \\
196 \\
196 \mathrm{~m} \\
198 \\
199\end{array}$ & $\begin{array}{c}\text { Half Life } \\
3.2 \mathrm{~h} \\
4.1 \mathrm{~h} \\
15.8 \mathrm{~h} \\
39.5 \mathrm{~h} \\
183 \mathrm{~d} \\
6.18 \mathrm{~d} \\
9.7 \mathrm{~h} \\
2.7 \mathrm{~d} \\
3.15 \mathrm{~d}\end{array}$ & $\begin{array}{c}\text { Decay } \\
\gamma, \mathrm{e}^{-} \\
\gamma, \mathrm{e}^{-}, \beta^{+} \\
\gamma, \mathrm{e}^{-} \\
\beta^{+}, \mathrm{e}^{-}, \gamma \\
\gamma, \mathrm{e}^{-} \\
\beta^{-}, \mathrm{e}^{-}, \gamma \\
\gamma, \mathrm{e}^{-} \\
\gamma, \mathrm{e}^{-} \\
\beta^{-}, \gamma, \mathrm{e}^{-}\end{array}$ & 5 & a \\
\hline Magnetic moment, $\mathrm{N} \times \mathrm{m}$ & & 0.1439 & & 6 & \\
\hline Spin & & $3 / 2$ & & 6 & \\
\hline $\begin{array}{l}\text { Thermal neutron capture } \\
\text { cross section, } \mathrm{cm}^{2}\end{array}$ & & $99 \times 10^{24}$ & & 7 & b \\
\hline
\end{tabular}

(a) At least 26 unstable isotopes of gold have been identified. Only those with half lives of more than one hour have been included in this tabulation.

(b) Value for formation of gold-198. See (2), 94-96, for the energy dependence of capture cross section for low energy neutrons.

Atomic and Crystallographic Properties $(1,2)$

\begin{tabular}{|c|c|c|c|c|}
\hline & Numerical value & Conditions & Ref. & Notes \\
\hline Atomic weight, $\mathrm{g}$ /atom & 196.9665 & & 8 & \\
\hline Electronic configuration & {$[X e] 4 f^{14} 5 d^{10} 6 s^{1}$} & & 9 & \\
\hline $\begin{array}{l}\text { Magnetic moment, Bohr's } \\
\text { magneton }\end{array}$ & 1.73 & & 10 & \\
\hline Atomic radius, $\mathrm{cm}$ & $1.442 \times 10^{-8}$ & Gold lattice & 11 & \\
\hline Electronegativity, $\mathrm{V}$ & 2.4 & & 8 & a \\
\hline Crystal structure & F.c.c. & & & \\
\hline Space group & $\mathrm{Fm} 3 \mathrm{~m}$ & & & \\
\hline Lattice constant, $\mathrm{cm}$ & $4.07 \times 10^{-8}$ & At room temp. & & $\mathrm{b}$ \\
\hline Inter-atomic distance, $\mathrm{cm}$ & $2.878 \times 10^{-8}$ & At room temp. & 12 & \\
\hline Morphology & Cubic-holohedral & & & \\
\hline Usual habitus & Octahedral or dodecahedral & & & \\
\hline $\begin{array}{l}\text { Temperature of } \\
\text { recrystallisation, } \mathrm{K}\end{array}$ & $<373$ & & 13 & $\mathrm{c}$ \\
\hline
\end{tabular}

(a) According to W. Gordy (42) the value of the electronegativity would be $3.1 \mathrm{~V}$.

(b) Average value from more than 50 references.

(c) The temperature of actuat recrystallisation is dependent on time of exposure; 'Tamman' temperature: $445 \mathrm{~K}$. 
Mechanical Properties $(1,2,3)$

\begin{tabular}{|c|c|c|c|c|}
\hline & Numerical value & Conditions & Ref. & Notes \\
\hline Density, $\mathrm{g} / \mathrm{cm}^{3}$ & 19.32 & At $293 \mathrm{~K}$ & & $\mathrm{a}$ \\
\hline $\begin{array}{l}\text { Hardness, } \mathrm{kg} / \mathrm{mm}^{2} \\
\text { BHN }\end{array}$ & $\begin{array}{l}25 \\
43 \\
52 \\
58\end{array}$ & $\begin{array}{l}\text { Annealed at } 1013 \mathrm{~K} \\
\text { After } 15 \% \text { reduction } \\
\text { After } 30 \% \text { reduction } \\
\text { After } 60 \% \text { reduction }\end{array}$ & 14 & \\
\hline DPN & $\begin{array}{l}25 \text { to } 27 \\
55 \text { to } 60 \\
33 \text { to } 35\end{array}$ & $\begin{array}{c}\text { Annealed at } 573 \mathrm{~K} \\
\text { After } 60 \% \text { reduction } \\
\text { As cast }\end{array}$ & 15 & b \\
\hline $\begin{array}{l}\text { Modulus of elasticity, } \\
\text { GPa }\end{array}$ & 79 & $\begin{array}{c}\text { At } 293 \mathrm{~K} \\
\text { Annealed at } 1173 \mathrm{~K}\end{array}$ & 16 & \\
\hline Poisson's ratio & 0.42 & As drawn & 17 & \\
\hline Tensile strength, $\mathrm{MPa}$ & $\begin{array}{c}235 \\
124 \text { to } 137 \\
206 \text { to } 221\end{array}$ & $\begin{array}{l}\text { Theoretical value } \\
\text { Annealed at } 573 \mathrm{~K} \\
60 \% \text { cold worked }\end{array}$ & $\begin{array}{l}18 \\
15\end{array}$ & c \\
\hline $\begin{array}{l}\text { Elongation, per cent in } \\
50 \mathrm{~mm}\end{array}$ & $\begin{array}{c}39 \text { to } 45 \\
4\end{array}$ & $\begin{array}{l}\text { Annealed at } 573 \mathrm{~K} \\
60 \% \text { cold worked }\end{array}$ & 15 & d \\
\hline Compressibility, $\mathrm{m}^{2} / \mathrm{N}$ & $6.01 \times 10^{-12}$ & At $300 \mathrm{~K}$ & 19 & e \\
\hline Internal friction $\mathrm{g} / \mathrm{cm} / \mathrm{s}$ & $17 \times 10^{8}$ & & 20 & \\
\hline
\end{tabular}

(a) Commercially accepted value. Pyknometric determinations at room temperature have vielded values between 19.21 and $19.93 \mathrm{~g} / \mathrm{cm}^{3}$. X-ray diffraction determinations have yielded values between 19.29 and $19.37 \mathrm{~g} / \mathrm{cm}^{3}$. See (1), 451-452.

(b) Hardness measured on $0.5 \mathrm{~mm}$ diameter wire, except 'as cast' which was measured on rod.

(c) Tensile strength and elongation measured on $0.5 \mathrm{~mm}$ diameter wire.

(d) According to (46) the mean value of compressibility to $1.22 \mathrm{GPa}$ at $303 \mathrm{~K}$ is $5.3 \times 10^{-12} \mathrm{~m}^{2} / \mathrm{N}$.

Thermal Properties $(1,3,4)$

\begin{tabular}{|c|c|c|c|c|}
\hline & Numerical value & Conditions & Ref. & Notes \\
\hline Melting point, $\mathrm{K}$ & 1337.59 & & & a \\
\hline Heat of fusion, J/g-atom & $1.268 \times 10^{4}$ & & 21 & \\
\hline Boiling point, $\mathrm{K}$ & 3081 & & 22 & \\
\hline $\begin{array}{l}\text { Heat of evaporation, } \\
\mathrm{J} / \mathrm{g} \text {-atom }\end{array}$ & $3.653 \times 10^{5}$ & At $298 \mathrm{~K}$ & 23 & \\
\hline Vapour pressure, $\mathrm{Pa}$ & $\begin{array}{l}5.5 \times 10^{-8} \\
8.5 \times 10^{-2} \\
8.2 \times 10^{1} \\
4.9 \times 10^{3} \\
7.1 \times 10^{5}\end{array}$ & $\begin{array}{l}\text { At } 1000 \mathrm{~K} \\
\text { At } 1500 \mathrm{~K} \\
\text { At } 2000 \mathrm{~K} \\
\text { At } 2500 \mathrm{~K} \\
\text { At } 3000 \mathrm{~K}\end{array}$ & 23 & \\
\hline Specific heat, $\mathrm{J} / \mathrm{g} \times \mathrm{K}$ & $1.288 \times 10^{-1}$ & At $298 \mathrm{~K}$ & 22 & $b$ \\
\hline $\begin{array}{l}\text { Characteristic } \\
\text { temperature, } \mathrm{K}\end{array}$ & 178 & & 24 & \\
\hline $\begin{array}{l}\text { Thermal conductivity, } \\
\mathrm{W} / \mathrm{cm} \times \mathrm{K}\end{array}$ & 3.144 & At $273 \mathrm{~K}$ & 25 & \\
\hline Thermal expansion, $\mathrm{K}^{-1}$ & $1.416 \times 10^{-7}$ & From 273 to $373 \mathrm{~K}$ & 26 & $c$ \\
\hline
\end{tabular}

(a) Calibration point for International Practical Temperature Scale (1968).

(b) Heat capacity between 15 and $300 \mathrm{~K}$ : see (43); between 273 and 1336: see (44).

(c) Interpolation formula for 273 to $1173 \mathrm{~K}: \alpha_{o, t}=14.06 \times 10^{-6}+1.672 \times 10^{-9} \mathrm{t}+1.197 \times 10^{-12} \mathrm{t}^{2}$, (t in $\left.{ }^{\circ} \mathrm{C}\right),(45)$. 
Electrical Properties $(1,2,3)$

\begin{tabular}{|l|c|c|c|c|}
\hline & Numerical value & Conditions & Ref. & Notes \\
\cline { 2 - 5 } $\begin{array}{l}\text { Electrical resistivity, } \\
\Omega \times \mathrm{cm}\end{array}$ & $2.05 \times 10^{-5}$ & At $273 \mathrm{~K}$ & 27 & $\mathrm{a}$ \\
$\begin{array}{l}\text { Temperature coefficient } \\
\text { of resistivity, } \mathrm{K}^{-1}\end{array}$ & $4.06 \times 10^{-3}$ & From 273 to $373 \mathrm{~K}$ & 27 & $\mathrm{a}, \mathrm{b}$ \\
Contact resistance, & $6 \times 10^{-9}$ & At room temperature & 28 & $\mathrm{c}$ \\
$\Omega / \mathrm{cm}^{2}$ & 7.69 to $7.85 \times 10^{-19}$ & Thermionic & 29 & \\
Work function, J & 8.17 to $8.76 \times 10^{-19}$ & Photoelectric & 30 & \\
lonization potential, $V$ & 9.2 & Au $\rightarrow \mathrm{Au}^{+}$ & 31 & \\
Thomson effect, & 20.5 & Au $\rightarrow \mathrm{Au}^{2+}$ & 31 & \\
J/C $\times \mathrm{K}$ & 1.76 & At $298 \mathrm{~K}$ & 32 & \\
Thermal EMF vs. & & At $373 \mathrm{~K}$ & 15 & $\mathrm{~d}$ \\
N.B.S. Pt 27, mV & +0.92 & At $773 \mathrm{~K}$ & & \\
& +12.35 & At $1073 \mathrm{~K}$ & & \\
\hline
\end{tabular}

(a) The electrical resistivity, particularly its temperature dependence, is affected by mechanical and thermal pretreatment, presence of impurities, pressure, magnetic fields, it increases in thin layers and decreases by illumination of thin layers.

(b) Temperature dependence of the resistivity $(27):-0.1982+8.3123 \times 10^{-3} \mathrm{~T}-0.7091 \times 10^{-6} \mathrm{~T}^{2}+1.4795 \times 10^{-9} \mathrm{~T}^{3}$.

(c) Average value for loads from 1 to $400 \mathrm{~g}$.

(d) Cold junction at ice point. N.B.S. Pt 27 is U.S. Standard material derived from platinum melt No. 27 of the National Bureau of Standards. For the temperature function of thermoelectric power, see (27).

Optical Properties $(1,2,4)$

\begin{tabular}{|c|c|c|c|c|c|c|}
\hline \multirow{2}{*}{$\begin{array}{l}\text { Reflectance, per } \\
\text { cent; refraction } \\
\text { and extinction } \\
\text { coefficient }\end{array}$} & \multicolumn{3}{|c|}{ Numerical value } & \multirow{2}{*}{$\begin{array}{c}\text { Conditions } \\
\\
\\
\\
\lambda, \mu \mathrm{m} \\
\\
0.40 \\
0.45 \\
0.50 \\
0.55 \\
0.60 \\
0.70 \\
0.80 \\
0.90\end{array}$} & \multirow{2}{*}{$\begin{array}{c}\text { Ref. } \\
33,34\end{array}$} & \multirow{2}{*}{ Notes } \\
\hline & $\begin{array}{c}\text { Reflec- } \\
\text { tance } \\
38.7 \\
38.7 \\
47.7 \\
81.6 \\
91.9 \\
96.7 \\
97.4 \\
97.8 \\
98.1\end{array}$ & $\begin{array}{c}\text { Refr. } \\
\text { ind. } \\
- \\
- \\
- \\
0.331 \\
0.200 \\
0.131 \\
0.149 \\
0.166 \\
0.179\end{array}$ & $\begin{array}{c}\text { Ext. } \\
\text { coef. } \\
- \\
- \\
- \\
2.324 \\
2.897 \\
3.842 \\
4.654 \\
5.335 \\
6.044\end{array}$ & & & \\
\hline Total emissivity & \multicolumn{3}{|c|}{0.018 to 0.035} & From 493 to $893 \mathrm{~K}$ & 35 & \\
\hline Spectral emissivity & \multicolumn{2}{|l|}{$\begin{array}{r}\lambda, m \\
71 \\
2 \\
5 \\
10 \\
15\end{array}$} & $\begin{array}{c}\text { nittance, \% } \\
7 \\
10 \\
6 \\
6 \\
6\end{array}$ & At $973 \mathrm{~K}$ & 36 & $a, b$ \\
\hline Arc spectrum & $\begin{array}{c}\lambda, \mu \mathrm{m} \\
0.267595 \\
0.242795\end{array}$ & $\begin{array}{c}\text { Excit. pot. } \\
4.6 \\
5.1\end{array}$ & $\begin{array}{l}\text { Rel. int. } \\
250 \mathrm{R} \\
400 \mathrm{R}\end{array}$ & & 37 & c \\
\hline X-ray emission, $\mathrm{nm}$ & $\begin{array}{c}\alpha_{1} \\
\text { K } 0.0180 \\
\text { L } 0.1276 \\
\text { M } 0.5827\end{array}$ & $\begin{array}{l}2 \\
4 \\
0\end{array}$ & $\begin{array}{l}\alpha_{2} \\
1851 \\
2878 \\
8372\end{array}$ & & 38 & \\
\hline
\end{tabular}

(a) Defined as the ratio of radiation of a gold surface to that of a black body at given temperature and wavelength.

(b) Measured on evaporated gold film.

(c) The two principal (most sensitive) lines of the arc spectrum have been listed. The letter $\mathrm{R}$ denotes wide self-reversal. 
Magnetic Properties $(1,2)$

\begin{tabular}{|c|c|c|c|}
\hline & Numerical value & Conditions & Ref. \\
\hline $\begin{array}{l}\text { Susceptibility, } \\
\text { cgs emu/g }\end{array}$ & $-0.143 \times 10^{-6}$ & At $291 \mathrm{~K}$ & 39 \\
\hline $\begin{array}{l}\text { Hall coefficient, } \\
\Omega \times \mathrm{cm} / \text { Gauss }\end{array}$ & $-6.97 \times 10^{-13}$ & At $295 \mathrm{~K}$ & 29 \\
\hline
\end{tabular}

Chemical Properties (1,4)

\begin{tabular}{|c|c|c|c|c|}
\hline & Numerical value & Conditions & Ref. & Notes \\
\hline Valence & 1,3 & & & \\
\hline Entropy, J/g-atom $\times \mathrm{K}$ & 47.33 & At $298 \mathrm{~K}$ & 40 & \\
\hline Chemical constant & 1.853 & & 24 & \\
\hline $\begin{array}{l}\text { Standard oxidation } \\
\text { potential, } V\end{array}$ & ca. $\begin{array}{r}-1.68 \\
-1.50\end{array}$ & $\begin{array}{c}\mathrm{Au} \rightarrow \mathrm{Au}^{+}+\mathrm{e} \\
\mathrm{Au} \rightarrow \mathrm{Au}^{3}+3 \mathrm{e}\end{array}$ & $\begin{array}{l}41 \\
41\end{array}$ & a \\
\hline
\end{tabular}

(a) Potential of 1 g-atom/I vs. standard hydrogen electrode.

\section{References}

1 'Gmelins Handbuch der Anorganischen Chemie', 8th Edition, System-Nummer 62, Part 3, Verlag Chemie, GmbH., Weinheim, 1954

2 'Gold: Recovery, Properties and Applications', edited by E. M. Wise, D. Van Nostrand Co., Inc., Princeton, N.J., 1964

3 G. Dubois in 'Monographie sur les Métaux de Haute Pureté, edited by G. Chaudron, Masson, Paris, 1977

4 R. Hultgren, R. L. Orr, P. D. Andersen and K Kelley, 'Selected Values of Thermodynamic Properties of Metals and Alloys', John Wiley \& Sons, Inc., New York, 1963

5 'Lange's Handbook of Chemistry', 11th Edition, McGraw-Hill Book Co., New York, 1973, 3, 92-94*

6 See (5), 3, 12*

$7 \mathrm{See}(5), 3,93^{\star}$

8 'CRC Handbook of Chemistry and Physics', 58th Edition, CRC Press, Cleveland, Ohio, 1977-1978, B-1

9 See (5), 3, 3*

10 T. Kitagawu, Rev. Phys. Chem. Fpn., 1937, 11, 71

11 See (5), 3, 120*

12 M. C. Neuburger, Z. Kristallogr., 1936, 93, 1

13 T. K. Rose, f. Inst. Met., 1912, 8, 86

14 L. Sterner Rainer, Z. Metallkd., 1926, 18, 143

15 Engelhard Industries Division, Engelhard Minerals \& Chemicals Corp:

16 W. Köster, Z. Metallkd, 1948, 39,

17 E. Grüneisen, Ann. Phys., 1908, 25, (4), 825

18 R. Fürth, Nature, 1940, 145, 741

19 Y. A. Chang and L. Himmel, J. Appl. Phys., 1966, 37, 3567

20 K. Jokibé and S. Sahai, Phil. Mag., 1921, 42, (6), 397

21 See (8), B-266

22 See (4), 39

23 See (4), 41

24 A. C. Egerton, Proc. Phys. Soc., 1924-1925, 37, 75
25 See (5), 10, 272*

$26 \mathrm{See}(2), 73$

27 M. J. Laubitz, Can. 7. Phys., 1969, 47, 2633

28 R. Holm, Z. Tech. Phys., 1931, 12, 663

29 See (2), 85

30 See (8), E-81

31 J. R. Platt and R. A. Sawyer, Phys. Rev., 1941, 60, (2), 866

32 G. Borelius, W. H. Keesom, C. H. Johansson and J. O. Linde, Proc. Acad. Sci., Amsterdam, 1930, 33, 17

33 See (2), 80

34 K. Weiss, Z. Naturforsch., 1948, 3a, 143

35 'IR Handbook', Svenska A. B. Philips Ljusavdelning, Stockholm, L1315, 68

36 E. A. Schatz and L. H. McCandless, 'Research for Low and High Emittance Coatings', Technical Documents, Report ASD TR 62 443, 1962, 13

37 G. R. Harrison, 'Wavelength Tables', The M.I.T. Press, Cambridge, Mass., 1969, XXII

38 'X-Ray Emission Line Wave Length and Two Theta Tables', ASTM Data Series DS 37, Am. Soc. Test. Mater., Philadelphia, 1965, 1-100; M. F. R. Hirsh, Phys. Rev., 1931, 38, (2), 914

39 C. M. Hurd, F. Phys. Chem. Solids, 1966, 27, 1371

40 K. K. Kelley and E. G. King, Bull. Bur. Mines, $1961,592,43$

41 W. H. Latimer, 'The Oxidation Status of the Elements and Their Potentials in Aqueous Solutions', Prentice Hall, New York, 1952, 195

42 W. Gordy, 7. Chem. Phys, 1946, 14, 305

43 T. H. Geballe and W. F. Giauque, f. Am. Chem. Soc., 1952, 74, 2368

44 K. K. Kelley, Bull. Bur. Mines, 1934, 371

45 J. B. Austin, Physics, 1932, 3, 240

46 P. W. Bridgman, Proc. Nat. Acad. Sci. Washington, $1922,8,361$

«Used with permission of McGraw-Hill Book Company 